\title{
Significance of Ebp1 and p53 protein expression in cervical cancer
}

\author{
L. Liu ${ }^{1 *}$, X.D. Li ${ }^{2 *}$, H.Y. Chen ${ }^{1}$, J.S. Cui ${ }^{1}$ and D.Y. Xu ${ }^{2}$ \\ ${ }^{1}$ Department of Pathology, Affiliated Hospital of Yanbian University, Yanji, Jilin, China \\ ${ }^{2}$ Center of Morphological Experiment, Medical College of Yanbian University, Yanji, \\ Jilin, China
}

*These authors contributed equally to this study.

Corresponding author: D.Y. Xu

E-mail: dongyuanx_ybu@126.com

Genet. Mol. Res. 14 (4): 11860-11866 (2015)

Received April 23, 2015

Accepted July 1, 2015

Published October 2, 2015

DOI http://dx.doi.org/10.4238/2015.October.2.19

ABSTRACT. In this study, the ErbB3-binding protein (Ebp1) and p53 protein expression in cervical cancer tissues, and its significance in the prognosis of the disease was investigated. Ebp1 and p53 protein expression was detected by immunohistochemical analysis in cervical cancer tissues $(\mathrm{N}=60)$ and normal tissues adjacent to the cancer tissues $(\mathrm{N}=60)$. The rates of positive Ebp1 and p53 protein expression were 35.0 and $60.0 \%$, respectively. Ebp1 and p53 were overexpressed in cervical cancer tissues, compared to normal tissues $(P<0.05)$. Ebp1 and p53 protein expression was not correlated with age, tumor size, or family tumor history $(P>0.05)$. However, high levels of expression of Ebp1 and p53 were positively correlated with the TNM stage and lymphatic metastasis in cervical cancer patients $(P<0.05)$. The combined determination of Ebp1 and p53 expression levels in cervical cancer patients could support the effective prediction of metastatic potential and patient prognosis.

Key words: Ebp1; p53; Cervical cancer; Immunohistochemistry 


\section{INTRODUCTION}

Cervical cancer is the second most common gynecological malignant tumor affecting women (Boyle and Ferlay, 2005). Studies indicate that cervical cancer develops as a result of the combined action of many genes. Malignant cellular transformation that can lead to cancer is caused by oncogene and antioncogene dysfunction, occurring as a result of the effects of multiple carcinogenic environmental factors. ErbB3-binding protein (Ebp1) is a member of the PA2G4 family of proliferation-regulated proteins that is ubiquitously expressed in the cytoplasm and nucleus of multiple malignant and non-malignant cells (Yoo et al., 2000; Xia et al., 2001). A few recent studies have described the expression of Ebp1 in tumor tissues, and its clinicopathological relevance. A recent publication has also revealed an overexpression of Ebp1 in prostate and colorectal cancers, compared to the normal areas adjacent to the cancer (Santegoets et al., 2007; Gannon et al., 2008).

A well-known abnormal gene expression pattern in cervical cancer is p53 degradation. Unlike most solid tumors, the p53 mutation is infrequently found in cervical cancer. Interestingly, human glioblastoma studies have revealed Ebp1 to be one of the many p53 target genes (Kim et al., 2010). So far, the correlation between the combined expression of $p 53$ and Ebp1 in cervical cancer tissue and the metastasis potential and patient prognosis has not been reported. In this study, immunohistochemical methods were adopted to measure the protein expression levels of p53 and Ebp1 in cervical cancer tissues, and to determine their relationship with the clinicopathological features of the disease.

\section{MATERIAL AND METHODS}

\section{Specimens}

Sixty tumor samples surgically resected from cervical cancer patients admitted to the Yanbian University Hospital between November 2004 and February 2013 served as the experimental group, and 60 tissue samples collected from normal tumor-adjacent regions from the cervical cancer patients served as the control group. The age of the patients ranged from 33 to 74 years old. The tumor diameter was $\leq 5 \mathrm{~cm}$ in 38 samples and $>5 \mathrm{~cm}$ in 22 samples. Thirty four of the 60 cervical cancer patients were in clinical stages I and II, 26 were in stage III, and 28 were diagnosed with lymph node metastases. Postoperative pathological diagnosis confirmed that all patients suffered from cervical cancer. This study was conducted in accordance with the Declaration of Helsinki, and with approval from the Ethics Committee of the Medical College of Yanbian University.

\section{Experimental methods}

The samples were subjected to streptavidin-peroxidase immunohistochemical analysis. The sample sections were de-paraffinized with dimethyl benzene and dehydrated with an ethanol gradient series; antigen was retrieved by boiling the tissue sections in $0.01 \mathrm{M}$ citrate buffer, $\mathrm{pH}$ 6.0 , for $10 \mathrm{~min}$, and subsequently naturally cooling them. Endogenous peroxidase activity was inactivated using $3 \% \mathrm{H}_{2} \mathrm{O}_{2}$, and the tissue sections were rinsed thrice with phosphate buffered saline (PBS). Subsequently, the sections were incubated in a protein blocking serum-free reagent (Dako, Carpinteria, CA, USA) at room temperature for $15 \mathrm{~min}$, in order to block the non-specific staining. Thereafter, the sections were incubated with mouse anti-Ebp1 monoclonal antibody (1:100; Abcam 
Ltd., Cambridge, UK) or mouse anti-p53 monoclonal antibody (1:100; Beijing Zhongshan Golden Bridge Biotechnology Company, Beijing, China) overnight at $4^{\circ} \mathrm{C}$. The sections were washed in PBS, and incubated with biotin-conjugated goat anti-rabbit $\lg \mathrm{G}$ at $37^{\circ} \mathrm{C}$ for $2 \mathrm{~h}$. The samples were then incubated with the avidin-biotin complex solution for $1 \mathrm{~h}$ at room temperature. Finally, the sections were placed in 3,3'-diaminobenzidine tetrahydrochloride, and viewed and photographed with a light microscope (Nikon Eclipse 80i; Nikon, Tokyo, Japan). Sections provided by the reagent company were used as positive controls, and sections in which the primary antibody had been replaced by PBS were used as the negative control.

\section{Assessment and analysis}

The results were judged based on the percentage of positive cells and the degree of staining. The percentage of positive cells were assessed by random selection and observation of 5 high-power fields. The fields were scored 1, 2, 3, or 4 for $<10,10-50,51-75$, and $>75 \%$ positive cells, respectively. The degree of staining of the cells was judged by scoring $1,2,3$, or 4 , for cells showing no yellow coloration, faint yellow color, moderate yellow color, or brownish yellow coloration, respectively. The 2 scores were multiplied, and the total score was denoted as follows: 0 was denoted as (-), 1-3 was denoted as (+), 4-6 was denoted as (++), and 7-9 was denoted as $(+++) .(+++)$ was considered to be a strong positive result, $(+)$ and $(++)$ were considered to be positive results, and (-) was considered to be a negative result.

\section{Statistical analysis}

SPSS v.17.0 (IBM Corp., Armonk, NY, USA) was used for all statistical analyses. The chi-square test was used to compare the positive rates of Ebp1 and p53 expression between the tumor tissues and normal adjacent tissues. The correlation coefficients between Ebp1 and p53 were determined using a Pearson's rank correlation analysis. Differences with $\mathrm{P}$ value less than 0.05 were considered to be statistically significant.

\section{RESULTS}

\section{Ebp1 and p53 expression in cervical cancer and normal adjacent tissues}

Immunohistochemical staining showed that Ebp1 protein was mainly located in the cytoplasm or cell membrane; the positive region presented a faint yellow or brownish yellow color. The 553 protein was mainly located in the nucleus, with the positive region displaying brownish yellow particles. Among the 60 cervical cancer tissue samples, 36 were discovered to be p53positive (Figure 1A) and 24 were p53-negative (Figure 1B), for a p53-positive rate of 60.0\%. Ebp1 staining of the cervical cancer tissue samples showed that 21 cases were Ebp1-positive (Figure 1C), while 39 cases were Ebp1-negative (Figure 1D), for an Ebp1-positive rate of 35\%. Among the 60 normal adjacent tissue samples, 56 were p53-negative and 4 were p53-positive, for a p53positive rate of $6.7 \%$. Ebp1 staining of the normal tissue samples showed that 55 of the 60 samples were Ebp1-negative and 5 cases were Ebp1-positive, for an Ebp-1 positive rate of $8.3 \%$. The immunohistochemical staining showed that the protein expression levels of Ebp1 and p53 in the cervical cancer tissues were significantly higher than those in the normal adjacent tissues $(P<$ 0.05, Table 1). 

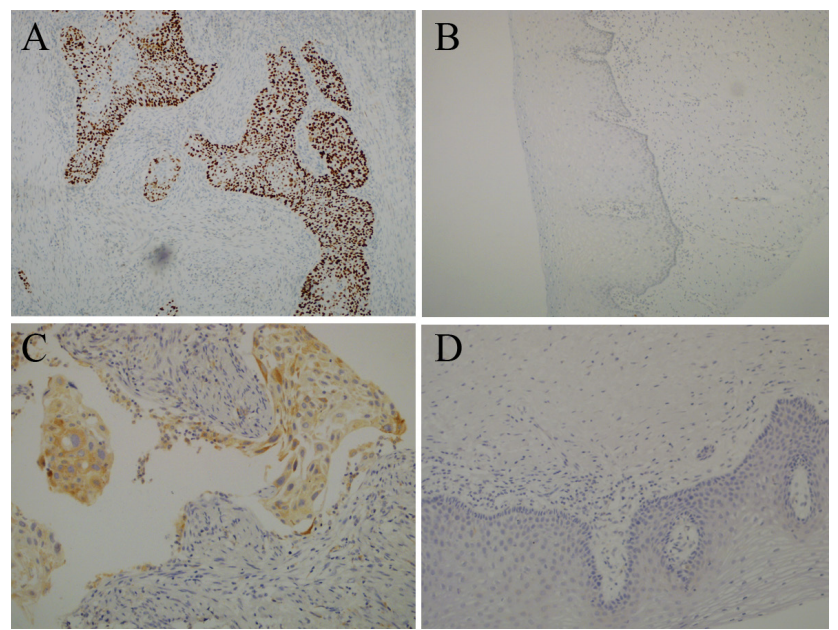

Figure 1. p53 and Ebp1 protein levels in cervical cancer and normal adjacent tissues. A. p53-positive cells in cervical cancer tissues (100X). B. p53-negative expression in cervical cancer tissues (100X). C. Ebp1-positive expression in cervical cancer tissues (200X). D. Ebp1-negative expression in cervical cancer tissue (200X).

Table 1. p53 and Ebp1 expression in cervical carcinoma tissues.

\begin{tabular}{lcccc}
\hline Index & \multicolumn{3}{c}{ Positive rate (\%) } & $X^{2}$ \\
\cline { 2 - 4 } & Tumor tissue & Normal adjacent & \\
\hline p53 & $60.0(36 / 60)$ & $6.7(4 / 60)$ & 38.4 & \\
Ebp1 & $35.0(21 / 60)$ & $8.3(5 / 60)$ & 12.57 & $<0.05$ \\
\hline
\end{tabular}

\section{Relationship between Ebp1 and p53 expression in cervical cancer tissues and the clinicopathological features of the tumor}

Ebp1 and p53 protein expression in cervical cancer tissue samples was not correlated with patient age, tumor size, or family history $(P>0.05$, Tables 2 and 3$)$. However, Ebp1 and p53 expression were both significantly associated with lymph node metastasis and the patient's cervical cancer TNM staging classification $(P<0.05)$.

\begin{tabular}{|c|c|c|c|c|c|c|c|c|}
\hline \multicolumn{2}{|c|}{ Clinicopathological features } & \multirow[t]{2}{*}{$\mathrm{N}$} & \multicolumn{4}{|c|}{ p53 } & \multirow[t]{2}{*}{ Positive rate (\%) } & \multirow[t]{2}{*}{$P$} \\
\hline & & & $(-)$ & $(+)$ & $(++)$ & $(+++)$ & & \\
\hline \multirow{2}{*}{ Age (years) } & $<60$ & 36 & 14 & 10 & 10 & 2 & 61.1 & \multirow{2}{*}{$>0.05$} \\
\hline & $\geq 60$ & 24 & 10 & 4 & 6 & 4 & 58.3 & \\
\hline \multirow[t]{2}{*}{ Tumor size $(\mathrm{cm})$} & $\leq 5$ & 38 & 16 & 8 & 10 & 4 & 57.9 & \multirow[t]{2}{*}{$>0.05$} \\
\hline & $>5$ & 22 & 8 & 6 & 6 & 2 & 63.6 & \\
\hline \multirow{2}{*}{ Family tumor history } & Positive & 18 & 6 & 2 & 6 & 4 & 66.6 & \multirow[t]{2}{*}{$>0.05$} \\
\hline & Negative & 42 & 18 & 10 & 12 & 2 & 57.1 & \\
\hline \multirow[t]{2}{*}{ TNM classification } & $I+I I$ & 34 & 20 & 6 & 6 & 2 & 41.2 & \multirow[t]{2}{*}{$<0.05$} \\
\hline & III & 26 & 4 & 8 & 10 & 4 & 84.6 & \\
\hline \multirow[t]{2}{*}{ Lymphatic metastasis } & Negative & 32 & 20 & 4 & 6 & 2 & 37.5 & \multirow[t]{2}{*}{$<0.05$} \\
\hline & Positive & 28 & 4 & 12 & 8 & 4 & 85.7 & \\
\hline
\end{tabular}




\begin{tabular}{|c|c|c|c|c|c|c|c|c|}
\hline \multicolumn{2}{|c|}{ Clinicopathological features } & \multirow[t]{2}{*}{$\mathrm{N}$} & \multicolumn{4}{|c|}{ Ebp1 } & \multirow[t]{2}{*}{ Positive rate (\%) } & \multirow[t]{2}{*}{$P$} \\
\hline & & & $(-)$ & $(+)$ & $(++)$ & $(+++)$ & & \\
\hline \multirow{2}{*}{ Age (years) } & $<60$ & 36 & 22 & 6 & 8 & 0 & 38.9 & $>0.05$ \\
\hline & $\geq 60$ & 24 & 17 & 2 & 3 & 2 & 41.2 & \\
\hline \multirow[t]{2}{*}{ Tumor size $(\mathrm{cm})$} & $\leq 5$ & 38 & 26 & 5 & 6 & 1 & 46.2 & $>0.05$ \\
\hline & $>5$ & 22 & 13 & 4 & 4 & 1 & 40.9 & \\
\hline \multirow{2}{*}{ Family tumor history } & Positive & 18 & 13 & 1 & 2 & 2 & 38.5 & $>0.05$ \\
\hline & Negative & 42 & 26 & 10 & 6 & 0 & 38.1 & \\
\hline \multirow[t]{2}{*}{ TNM } & $I+I I$ & 34 & 29 & 2 & 2 & 1 & 17.2 & $<0.05$ \\
\hline & III & 26 & 10 & 10 & 4 & 2 & 61.5 & \\
\hline \multirow[t]{2}{*}{ Lymphatic metastasis } & Negative & 32 & 27 & 2 & 2 & 1 & 18.5 & $<0.05$ \\
\hline & Positive & 28 & 12 & 6 & 8 & 2 & 57.1 & \\
\hline
\end{tabular}

\section{Correlation between Ebp1 and p53 expression}

The correlation coefficients between Ebp1 and p53 were determined using a Pearson's rank correlation test (Table 4). Among the 36 p53-positive cervical cancer cases, 19 were Ebp1positive and 17 were Ebp1-negative. Among the 24 p53-negative cervical cancer cases, 2 were found to be Ebp1-positive, while 22 were Ebp1-negative. Ebp1 and p53 protein expression were found to be significantly positively correlated $(r=0.456, \mathrm{P}<0.05)$.

\begin{tabular}{|c|c|c|c|c|c|}
\hline & \multicolumn{2}{|c|}{ Ebp1 } & \multirow[t]{2}{*}{ Total } & \multirow[t]{2}{*}{$r$} & \multirow[t]{2}{*}{$P$} \\
\hline & Positive & Negative & & & \\
\hline \multicolumn{6}{|l|}{ p53 } \\
\hline Positive & 19 & 17 & 36 & 0.456 & $<0.05$ \\
\hline Negative & 2 & 22 & 24 & & \\
\hline
\end{tabular}

\section{DISCUSSION}

Cervical cancer is a common malignant tumor of the female reproductive system, the incidence of which is second only to breast cancer. The aim of this study was to characterize Ebp1 expression in cervical cancer tissues, and to evaluate whether Ebp1 could represent a molecular marker capable of identifying patients at higher risk. Our results demonstrated a correlation between Ebp1 expression and cervical cancer. This data is consistent with previously published results demonstrating an overexpression of Ebp1 in prostate and colorectal cancer (Santegoets et al., 2007; Gannon et al., 2008). However, another study has shown a markedly higher expression of Ebp1 in tumor-adjacent tissues compared to carcinoma tissues (He et al., 2013). Previous reports have suggested that the anti-proliferative activity of Ebp1 is dependant on its nuclear localization (Squatrito et al., 2004). This finding of our study may be relevant to the two subtypes of Ebp1, p42 and p48, which have different effects on cell proliferation. The p48 Ebp1 subtype is mainly located in the cytoplasm and nucleus, and its overexpression promotes cell growth; on the other hand, the p42 Ebp1 subtype is mainly located in the cytoplasm, and its overexpression inhibits cell growth (Liu et al., 2006). p42 and p48 have different expression levels in different tissues, thereby regulating different functions, such as the growth and differentiation of tumor cells.

The wild-type and mutant $p 53$ genes are acknowledged anti-oncogenes. Mutant p53 is 
detectable by immunohistochemical methods, because of its stability and long half-life. Reports have revealed that the $p 53$ gene has a low mutation rate, but a high level of positive expression in cervical cancer tissues (Madhumati et al., 2012). Positive p53 expression is not indicative of mutation, but of the inclination for mutation, with the cancer cells growing rapidly once a p53 mutation occurs. Previous reports have suggested that the positive rate of p53 expression in cervical cancer tissue ranges from 32 to $67.8 \%$. Previous research has also demonstrated that ErbB-2 is synergistic with, or regulated by p53; the co-expression of these proteins can be used to predict highly malignant cervical cancer (Conesa-Zamora et al., 2013). Ebp1 partially inhibited the growth of ErbB-2/ErbB-3 expressing breast cancer cell lines by down-regulating the protein levels of ErbB2 (Zhang et al., 2008). Furthermore, Ebp1 p48 promoted the tumorigenicity of glioma cells through the down-regulation of p53. Cytoplasmic Ebp1 was found to be positively correlated with the nuclear expression p53, further supporting the role of Ebp1 in regulating p53 degradation. In this article, of the 36 cervical cancer cases with positive p53 expression, 21 cases displayed positive Ebp1 expression. High expression levels of p53 and Ebp1 were positively correlated with the TNM classification and lymph node metastasis, but not with the patient's family history, age, or tumor size. These results suggested that the Ebp1 protein expression tended to increase with the increase in positive p53 protein expression in cervical cancer tissues. This indicated that Ebp1 might be synergistic with, or regulated by, p53; the co-expression of Ebp1 and p53 was also deemed to be an indicator of a poor prognosis for cervical cancer patients.

p53 and Ebp1 play important roles in the development and metastasis of cervical cancer, and their synergistic effect promotes tumor cell infiltration and metastasis. Therefore, the combined detection of p53 and Ebp1 in cervical cancer could assist clinicians in judging the malignancy and metastasis rates, and supporting prevention, diagnosis, and treatment of cervical cancer.

\section{Conflicts of interest}

The authors declare no conflict of interest.

\section{ACKNOWLEDGMENTS} (\#2013-11).

Research supported by the Project of Education Department of the Jilin province of China

\section{REFERENCES}

Boyle P and Ferlay J (2005). Cancer incidence and mortality in Europe, 2004. Ann. Oncol. 16: 481-488.

Conesa-Zamora P, Torres-Moreno D, Isaac MA and Pérez-Guillermo M (2013). Gene amplification and immunohistochemical expression of ERBB2 and EGFR in cervical carcinogenesis. Correlation with cell-cycle markers and HPV presence. Exp. Mol. Pathol. 95: 151-155.

Gannon PO, Koumakpayi IH, Le Page C, Karakiewicz PI, et al. (2008). Ebp1 expression in benign and malignant prostate cancer. Cancer Cell Int. 8: 18.

He HC, Ling XH, Zhu JG, Fu X, et al. (2013). Down-regulation of the ErbB3 binding protein 1 in human bladder cancer promotes tumor progression and cell proliferation. Mol. Biol. Rep. 40: 3799-3805.

Kim CK, Nguyen TL, Joo KM, Nam DH, et al. (2010). Negative regulation of p53 by the long isoform of ErbB3 binding protein Ebp1 in brain tumors. Cancer Res. 70: 9730-9741.

Liu Z, Ahn JY, Liu X, and Ye K. (2006). Ebp1 isoforms distinctively regulate cell survival and differentiation. Proc. Natl. Acad. Sci. U. S. A. 103: 10917-10922.

Madhumati G, Kavita S, Anju M, Uma S, et al. (2012). Immunohistochemical expression of cell proliferating nuclear antigen 
(PCNA) and p53 protein in cervical cancer. J. Obstet. Gynaecol. India 62: 557-561.

Santegoets SJ, Schreurs MW, Reurs AW, Lindenberg JJ, et al. (2007). Identification and characterization of erbb-3-binding protein-1 as a target for immunotherapy. J. Immunol. 179: 2005-2012.

Squatrito M, Mancino M, Donzelli M, Areces LB, et al. (2004). EBP1 is a nucleolar growth-regulating protein that is part of preribosomal ribonucleoprotein complexes. Oncogene 23: 4454-4465.

Yoo JY, Wang XW, Rishi AK, Lessor T, et al. (2000). Interaction of the PA2G4 (EBP1) protein with ErbB-3 and regulation of this binding by heregulin. Br. J. Cancer 82: 683-690.

Xia X, Lessor TJ, Zhang Y, Woodford N, et al. (2001). Analysis of the expression pattern of Ebp1, an ErbB-3-binding protein. Biochem. Biophys. Res. Commun. 289: 240-244.

Zhang Y, Akinmade D and Hamburger AW (2008). Inhibition of heregulin mediated MCF-7 breast cancer cell growth by the ErbB3 binding protein EBP1. Cancer Lett. 265: 298-306. 